\title{
Asymmetric Annulation towards Pyrrolopiperazinones: Concise Enantioselective Syntheses of Pyrrole Alkaloid Natural Products
}

\author{
Barry M. Trost, ${ }^{*}$ Guangbin Dong \\ Department of Chemistry, Stanford University, Stanford, California 94305-5080. \\ bmtrost@stanford.edu
}

\section{Supporting Information}

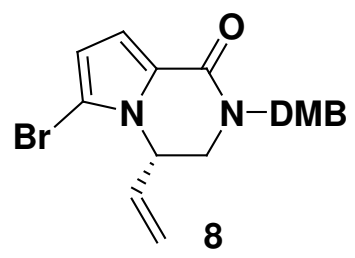

Compound 8: (eg. Table 1, entry 3) A solution of $\left[\mathrm{Pd}\left(\mathrm{C}_{3} \mathrm{H}_{5}\right) \mathrm{Cl}\right]_{2}(0.92 \mathrm{mg}, 0.0025 \mathrm{mmol})$ and $(R, R)-\boldsymbol{L}(5.2 \mathrm{mg}, 0.0075 \mathrm{mmol})$ in $0.5 \mathrm{ml}$ degassed DCM which had been stirring at rt for $10 \mathrm{~min}$, was added to a solution of compound $6(20.4 \mathrm{mg}, 0.1 \mathrm{mmol})$ and compound $7(22 \mathrm{mg}, 0.1 \mathrm{mmol})$ in DCM $(0.5 \mathrm{ml})$ under Ar. The mixture was stirred at $\mathrm{rt}$ for $16 \mathrm{hr}$, and then the solvent was removed under vacuum. Compound $\mathbf{8}$ with some uncyclized product were isolated via silica gel flash column chromatography (petroleum ether / ethyl acetate $=4 / 1$, then $3 / 2$ ) as a light yellow oil. After two days at $\mathrm{rt}$ under vacuum, the uncyclized intermediate was automatically converted to compound $\mathbf{8}$ as a light yellow foam. (28 mg, 72\%, 95\% ee by HPLC OD column, 90:10 heptane/i-propanol, 0.8 $\mathrm{ml} / \mathrm{min}$ ): $\mathrm{R}_{f}: 0.35$ (petroleum ether / ethyl acetate $\left.=3 / 2\right) ;[\alpha]_{\mathrm{D}}:-21.12(\mathrm{DCM}, \mathrm{c} 0.1) ;{ }^{1} \mathrm{H}$ NMR $\left(\mathrm{CDCl}_{3}, 500 \mathrm{MHz}\right) \delta 7.25(\mathrm{~m}, 1 \mathrm{H}), 6.95(\mathrm{~d}, J=4.0 \mathrm{~Hz}, 1 \mathrm{H}), 6.43(\mathrm{~m}, 2 \mathrm{H}), 6.26(\mathrm{~d}$, $J=4.0 \mathrm{~Hz}, 1 \mathrm{H}), 5.64(\mathrm{ddd}, J=17,10.5,5.0 \mathrm{~Hz}, 1 \mathrm{H}), 5.10(\mathrm{dd}, J=10.5,1.5 \mathrm{~Hz}, 1 \mathrm{H})$, $4.75(\mathrm{~m}, 2 \mathrm{H}), 4.60(\mathrm{~d}, J=17 \mathrm{~Hz}, 1 \mathrm{H}), 4.50$ (d, $J=14.5 \mathrm{~Hz}, 1 \mathrm{H}), 3.88$ (dd, $J=13,4.5 \mathrm{~Hz}$, $1 \mathrm{H}), 3.79(\mathrm{~s}, 6 \mathrm{H}), 3.48(\mathrm{dd}, J=13,1.5 \mathrm{~Hz}, 1 \mathrm{H}) ;{ }^{13} \mathrm{C}-\mathrm{NMR}\left(\mathrm{CDCl}_{3}, 125 \mathrm{MHz}\right) \delta 160.5$, $158.7,158.5,133.8,131.7,125.6,118.0,117.2,114.1,112.4,104.7,104.2,98.3,55.5$, 55.4, 54.6, 49.5, 43.7; IR (film) 2936, 1645, 1614, 1418, $1209 \mathrm{~cm}^{-1}$; HRMS $\left(\mathrm{C}_{18} \mathrm{H}_{19} \mathrm{BrN}_{2} \mathrm{O}_{3}\right)$ : Calc'd. $392.055858\left(\mathrm{M}^{+}\right)$, Found 392.056840.<smiles>CCN1C[C@H](CCO)n2c(Br)ccc2C1=O</smiles>

Compound 9: 9-BBN (99 $\mathrm{mg}, 0.40 \mathrm{mmol}$ ) was added to a solution of 8 (105 $\mathrm{mg}, 0.27$ $\mathrm{mmol})$ in THF $(2 \mathrm{ml})$ at $0{ }^{\circ} \mathrm{C}$ under $\mathrm{N}_{2}$. The resulting solution was stirred at $\mathrm{rt}$ for $3 \mathrm{~h}$, before it was quenched with $\mathrm{NaBO}_{3} \cdot \mathrm{H}_{2} \mathrm{O}(81 \mathrm{mg}, 0.81 \mathrm{mmol})$ and $\mathrm{H}_{2} \mathrm{O}(1 \mathrm{ml})$. The mixture was stirred at $\mathrm{rt}$ for $12 \mathrm{~h}$ before it was extracted with ethyl acetate $(20 \mathrm{ml} \times 3)$. The combined organic fraction was dried over $\mathrm{MgSO}_{4}$. Compound 9 was purified via 
silica gel flash column chromatography (5\% $\mathrm{MeOH}$ in DCM) to give a white foam (90 $\mathrm{mg}$, with $7.7 \mathrm{mg}$ starting material recovered, 82\%, brsm 88\%): $\mathrm{R}_{f}: 0.40(10 \% \mathrm{MeOH}$ in DCM); $[\alpha]_{\mathrm{D}}:-31.64$ (c 0.35, DCM); ${ }^{1} \mathrm{H}$ NMR $\left(\mathrm{CDCl}_{3}, 500 \mathrm{MHz}\right): \delta 7.28(\mathrm{~m}, 1 \mathrm{H}), 6.92$ $(\mathrm{d}, J=4.0 \mathrm{~Hz}, 1 \mathrm{H}), 6.45(\mathrm{~m}, 2 \mathrm{H}), 6.23(\mathrm{~d}, J=4.0 \mathrm{~Hz}, 1 \mathrm{H}), 5.00(\mathrm{~d}, J=14.5 \mathrm{~Hz}, 1 \mathrm{H})$, $4.42(\mathrm{~m}, 1 \mathrm{H}), 4.35(\mathrm{~d}, J=14 \mathrm{~Hz}, 1 \mathrm{H}), 3.81(\mathrm{~s}, 3 \mathrm{H}), 3.81(\mathrm{~s}, 3 \mathrm{H}), 3.77(\mathrm{~m}, 1 \mathrm{H}), 3.56(\mathrm{dd}, J$ $=13,1.5 \mathrm{~Hz}, 1 \mathrm{H}), 3.47(\mathrm{~m}, 1 \mathrm{H}), 3.33(\mathrm{~m}, 1 \mathrm{H}), 1.72(\mathrm{~m}, 2 \mathrm{H}) ;{ }^{13} \mathrm{C}-\mathrm{NMR}\left(\mathrm{CDCl}_{3}, 125 \mathrm{MHz}\right)$ $\delta 160.7,158.8,158.4,132.3,125.2,117.4,114.2,112.3,104.4,103.7,98.5,59.1,55.5$, 50.2, 47.6, 43.2, 35.0, 30.4; IR (film) 3383 (br), 2923, 2851, 1660, 1615, 1417, $1209 \mathrm{~cm}^{-1}$; HRMS $\left(\mathrm{C}_{18} \mathrm{H}_{21} \mathrm{BrN}_{2} \mathrm{O}_{4}\right)$ : Calc'd. $410.066422\left(\mathrm{M}^{+}\right)$, Found 410.068069 .

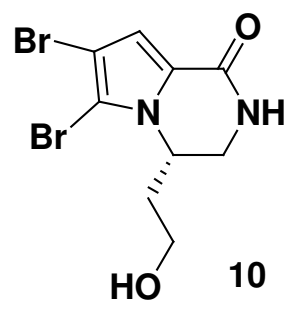

Compound 10: TFA $(0.6 \mathrm{ml})$ was added to a solution of $9(58 \mathrm{mg}, 0.14 \mathrm{mmol})$, tetrahydrothiophene $(59.4 \mathrm{mg}, 0.68 \mathrm{mmol})$ in DCM $(0.6 \mathrm{ml})$ at $\mathrm{rt}$. The resulting brown solution was stirred at $50{ }^{\circ} \mathrm{C}$ for $20 \mathrm{hr}$, before poured into pre-cooled sat. $\mathrm{NaHCO}_{3}$ solution. The mixture was extracted with ethyl acetate $(20 \mathrm{ml} \mathrm{x} \mathrm{3)}$, and the combined organic fraction was dried over $\mathrm{Na}_{2} \mathrm{SO}_{4}$. The products, as a ca. 2:1 mixture (monobromo-product and debromo-product) were purified via silica gel flash column chromatography $(10 \% \mathrm{MeOH} / \mathrm{DCM})$. The mixture was then dissolved with THF $(1 \mathrm{ml})$ and $\mathrm{MeOH}(0.5 \mathrm{ml})$. At $0{ }^{\circ} \mathrm{C}$, NBS $(37 \mathrm{mg}, 0.21 \mathrm{mmol})$ was added portionwisely to the above solution. The resulting solution was stirred at $\mathrm{rt}$ for $0.5 \mathrm{hr}$ before the solvents were removed under vacuum. Compound $\mathbf{1 0}$ was purified via silica gel flash column chromatography (5\% $\mathrm{MeOH}$ in DCM) to give a white foam $(46.1 \mathrm{mg}, 96 \%$ over two steps): $\mathrm{R}_{f}: 0.50\left(10 \% \mathrm{MeOH}\right.$ in ethyl acetate); $[\alpha]_{\mathrm{D}}:-27.3$ (c $\left.0.6, \mathrm{MeOH}\right) ;{ }^{1} \mathrm{H}$ NMR $\left(\mathrm{CD}_{3} \mathrm{OD}, 400 \mathrm{MHz}\right): \delta 6.92(\mathrm{~s}, 1 \mathrm{H}), 4.60(\mathrm{~m}, 1 \mathrm{H}), 3.80(\mathrm{dd}, J=13.6,4.0 \mathrm{~Hz}, 1 \mathrm{H}), 3.69-$ $3.64(\mathrm{~m}, 3 \mathrm{H}), 2.00(\mathrm{~m}, 1 \mathrm{H}), 1.85(\mathrm{~m}, 1 \mathrm{H}) ;{ }^{13} \mathrm{C}-\mathrm{NMR}\left(\mathrm{CD}_{3} \mathrm{OD}, 100 \mathrm{MHz}\right) \delta 161.1,126.1$, 116.4, 108.1, 101.3, 59.3, 53.3, 43.4, 35.5; IR (film) 3308 (br), 2927, 2882, 1652, 1550, 1465, 1429, $1338 \mathrm{~cm}^{-1}$; HRMS $\left(\mathrm{C}_{9} \mathrm{H}_{10} \mathrm{Br}_{2} \mathrm{~N}_{2} \mathrm{O}_{2}\right)$ : Calc'd. $335.910900\left(\mathrm{M}^{+}\right)$, Found 335.910485 .

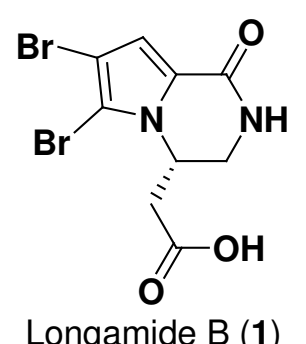

Method A: To $\mathrm{CH}_{3} \mathrm{CN}(0.5 \mathrm{ml})$ was added $\mathrm{H}_{5} \mathrm{IO}_{6}(17.6 \mathrm{mg}, 0.077 \mathrm{~mol})$ and the mixture was stirred vigorously at $\mathrm{rt}$ for $15 \mathrm{~min}$. A solution of compound $10(8.7 \mathrm{mg}, 0.026 \mathrm{mmol})$ in $\mathrm{CH}_{3} \mathrm{CN}(0.2 \mathrm{ml})$ was then added at $0{ }^{\circ} \mathrm{C}$, followed by addition of a $\mathrm{PCC}$ solution 


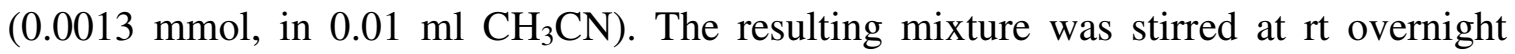
before diluted with ethyl acetate. The mixture was washed with brine, sat. aq. $\mathrm{NaHSO}_{3}$ and brine respectively. Longamide B (1) was further purified via a classical acid-base treatment as a white powder (5.7 mg, 63\%, brsm 90\%; $2.6 \mathrm{mg} \mathbf{1 0}$ was recovered).

Method B: TEMPO (0.44 mg, $0.0028 \mathrm{mmol})$ was added to a mixture of compound $\mathbf{1 0}$ (9.5 mg, $0.028 \mathrm{mmol}), \mathrm{PhI}(\mathrm{OAc})_{2}(22.7 \mathrm{mg}, 0.0705 \mathrm{mmol})$ and $\mathrm{NaHCO}_{3}(9.4 \mathrm{mg}, 0.112$ mmol) in DCM $(0.15 \mathrm{ml})$ at $\mathrm{rt}$ under air. The resulting solution was stirred at rt for $3 \mathrm{hr}$, before quenched with sat. $\mathrm{Na}_{2} \mathrm{~S}_{2} \mathrm{O}_{3}$ and sat. $\mathrm{NaHCO}_{3}$. The mixture was diluted with ethyl acetate, and longamide B (1) was further purified via a classical acid-base treatment as a white powder $(9.3 \mathrm{mg}, 95 \%)$.

mp 203-204 ${ }^{\circ} \mathrm{C}$ (decomposed to black tar); $[\alpha]_{D}:-8.21$ (c 0.52, MeOH); ${ }^{1} \mathrm{H}$ NMR $\left(\mathrm{CD}_{3} \mathrm{OD}, 500 \mathrm{MHz}\right): \delta 6.94(\mathrm{~s}, 1 \mathrm{H}), 4.81(\mathrm{~m}, 1 \mathrm{H}), 3.89(\mathrm{ddd}, J=13.5,4.5,1.5 \mathrm{~Hz}, 1 \mathrm{H})$, $3.66(\mathrm{dd}, J=13.5,1.5 \mathrm{~Hz}, 1 \mathrm{H}), 2.86(\mathrm{dd}, J=16.5,11 \mathrm{~Hz}, 1 \mathrm{H}), 2.55$ (ddd, $J=16.5,3.5$, $1.5 \mathrm{~Hz}, 1 \mathrm{H})$; IR: 3282, 3203, 2924, 1715, 1645, 1558, $1428 \mathrm{~cm}^{-1}$; HRMS $\left(\mathrm{C}_{9} \mathrm{H}_{8} \mathrm{Br}_{2} \mathrm{~N}_{2} \mathrm{O}_{3}\right)$ : Calc'd. $349.890164\left(\mathrm{M}^{+}\right)$, Found 349.889306.

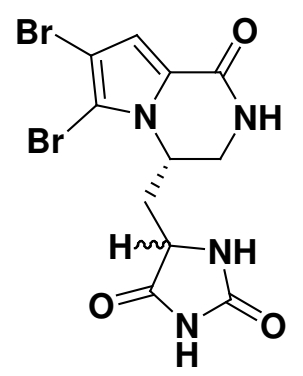

Agesamides A and B (5)

Dess-Martin periodane (34.3 $\mathrm{mg}, 0.081 \mathrm{mmol})$ was added to a mixture of compound $\mathbf{1 0}$ $(16.5 \mathrm{mg}, 0.040 \mathrm{mmol}), \mathrm{NaHCO}_{3}(10.1 \mathrm{mg}, 0.12 \mathrm{mmol})$ in $\mathrm{DCM}(0.4 \mathrm{ml})$ at $0{ }^{\circ} \mathrm{C}$. The resulting suspension was stirred at $\mathrm{rt}$ for $0.5 \mathrm{hr}$, before quenched with sat. $\mathrm{Na}_{2} \mathrm{~S}_{2} \mathrm{O}_{3}$ and sat. $\mathrm{NaHCO}_{3}$. The mixture was extracted with ethyl acetate $(10 \mathrm{ml} \times 3)$, and the combined organic fraction was dried over $\mathrm{Na}_{2} \mathrm{SO}_{4}$. The solvent was removed, and the resultant aldehyde with $\left(\mathrm{NH}_{4}\right)_{2} \mathrm{CO}_{3}(8.8 \mathrm{mg}, 0.1125 \mathrm{mmol})$ and $\mathrm{KCN}(3.0 \mathrm{mg}, 0.045 \mathrm{mmol})$ was then dissolved in $\mathrm{EtOH} / \mathrm{H}_{2} \mathrm{O}(1: 1,0.4 \mathrm{ml})$. The resulting solution was stirred vigorously at $60{ }^{\circ} \mathrm{C}$ overnight. The solvents were removed under vacuum, and agesamides $\mathrm{A}$ and $\mathrm{B}$ (5) was purified via preparative silica gel TLC (10\% $\mathrm{MeOH}$ in ethyl acetate) as a white solid (1:1 mixture, $17.5 \mathrm{mg}, 100 \%$ over two steps): ${ }^{1} \mathrm{H}$ NMR (DMSO- $d_{6}, 400 \mathrm{MHz}$ ): $\delta$ 10. $80(\mathrm{br}, 1 \mathrm{H}), 8.18(\mathrm{~s}, 1 \mathrm{H}), 8.04(\mathrm{~s}, 1 \mathrm{H}), 7.89-7.88(2 \mathrm{H}), 6.85(\mathrm{~s}, 2 \mathrm{H}), 4.65(\mathrm{~m}, 1 \mathrm{H})$, $4.53(\mathrm{~d}, J=11.2 \mathrm{~Hz}, 1 \mathrm{H}), 4.20(\mathrm{ddd}, J=11,3.0,1.5 \mathrm{~Hz}, 1 \mathrm{H}), 4.13(\mathrm{~m}, 1 \mathrm{H}), 3.78-3.70$ (2H), 3.54-3.46 (2H), 2.12 (ddd, $J=14,11.5,3.5 \mathrm{~Hz}, 1 \mathrm{H}$ ), 2.04 (ddd, $J=14.0,9.0,7.0$ $\mathrm{Hz}, 1 \mathrm{H}), 1.79(\mathrm{~m}, 1 \mathrm{H}), 1.53(\mathrm{~m}, 1 \mathrm{H}) ;{ }^{13} \mathrm{C}-\mathrm{NMR}$ (DMSO- $\left.d_{6}, 100 \mathrm{MHz}\right) \delta 175.2,174.9$, $157.53,157.46,157.2$, 157.1, 125.5, 114.0, 113.9, 105.71, 105.67, 99.3, 54.6, 54.3, 50.6, 41.7, 40.2, 33.8, 33.5; IR: 3228 (br), 2926, 2854, 1724, 1646, 1550, 1429, $1338 \mathrm{~cm}^{-1}$; HRMS $\left(\mathrm{C}_{11} \mathrm{H}_{10} \mathrm{Br}_{2} \mathrm{~N}_{4} \mathrm{O}_{3}\right)$ : Calc'd. $403.911963\left(\mathrm{M}^{+}\right)$, Found 403.910347. 


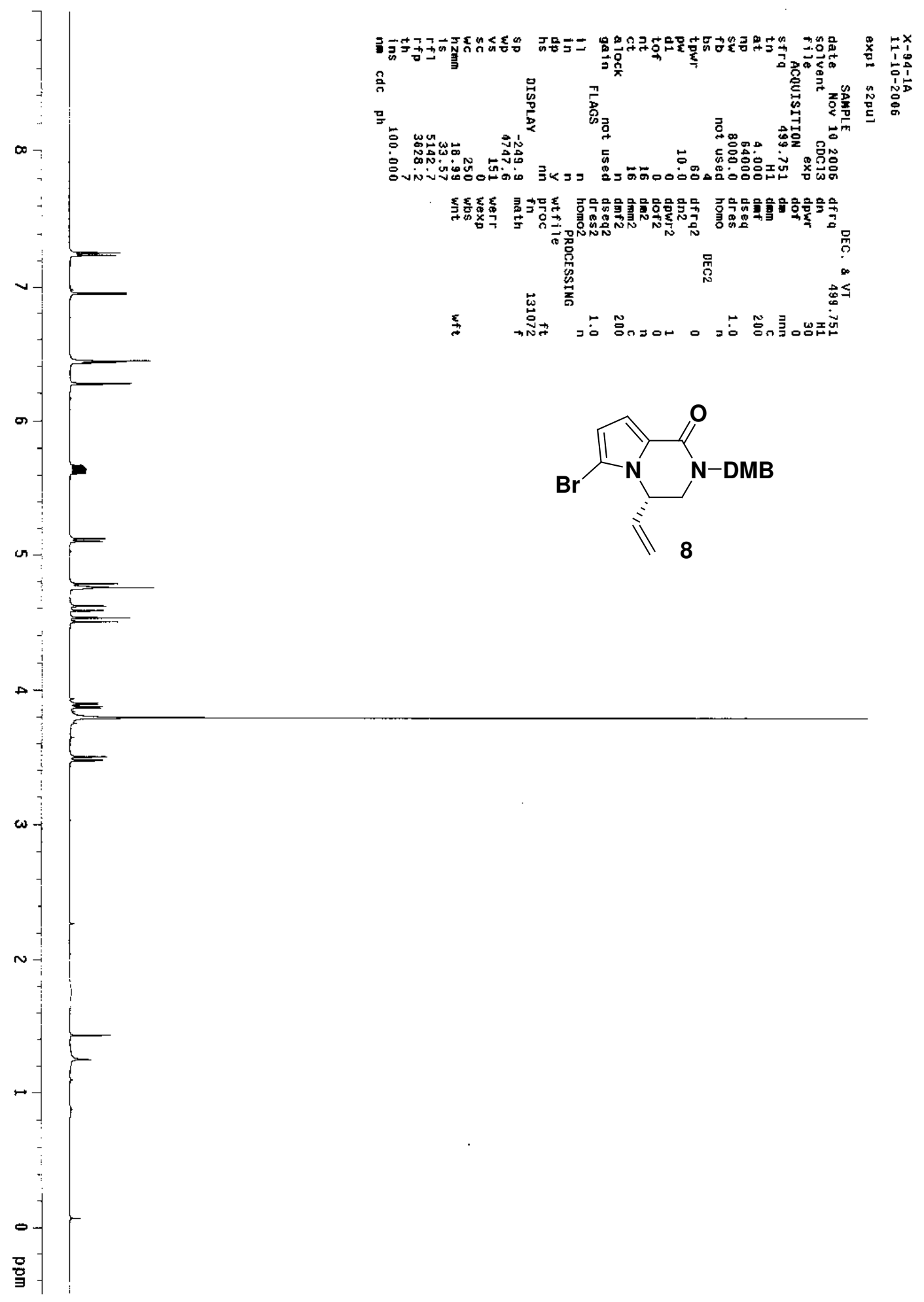




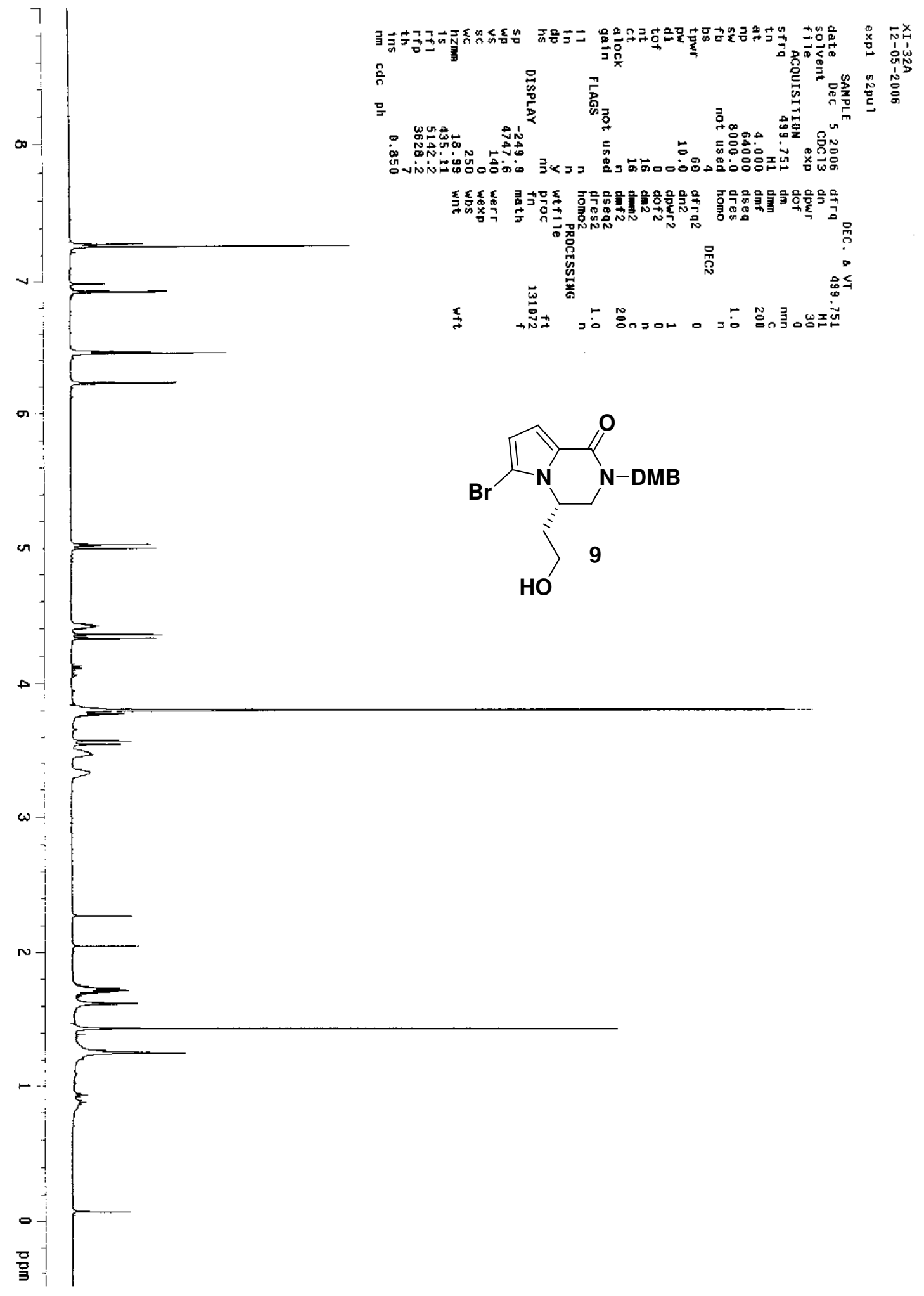




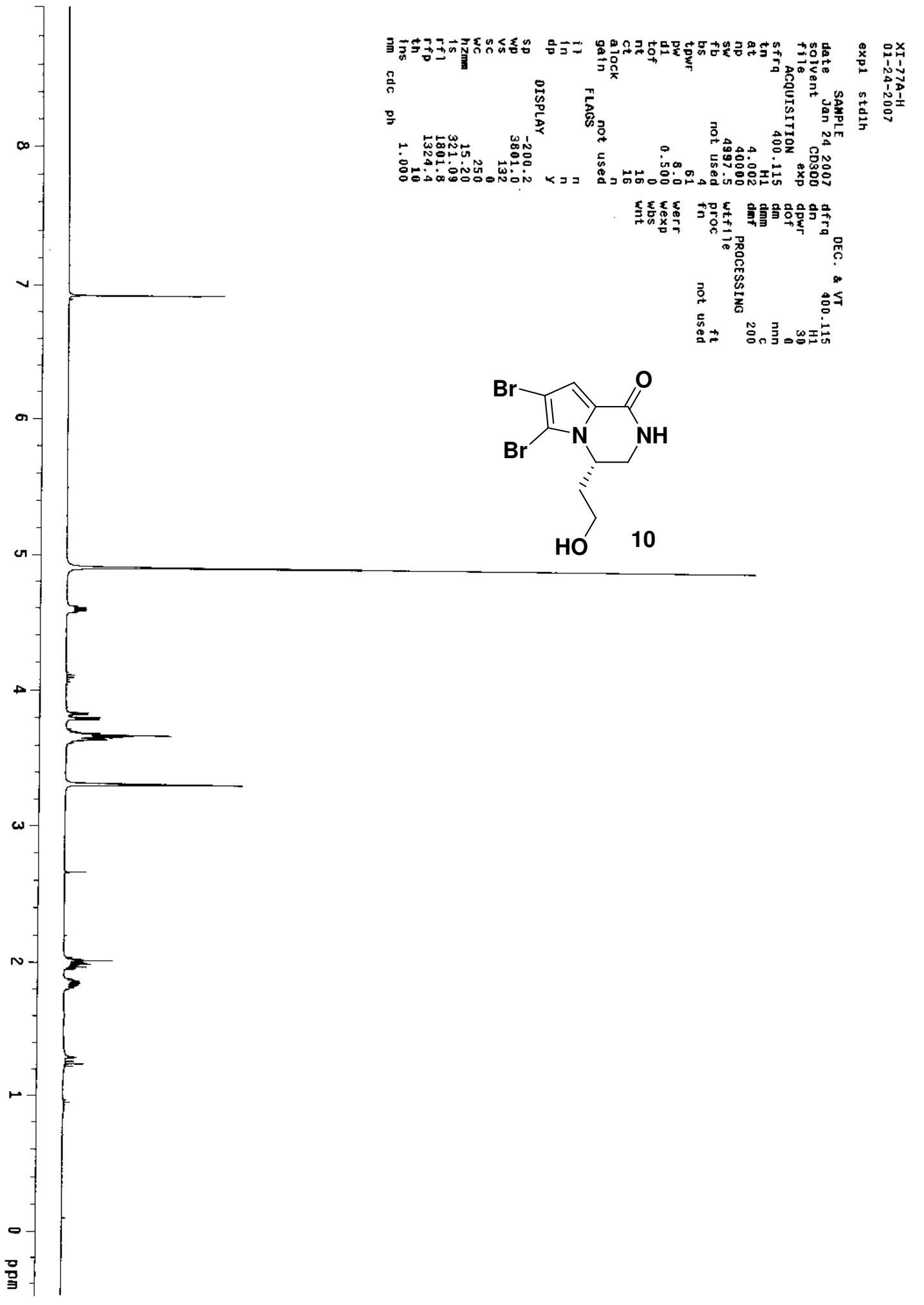




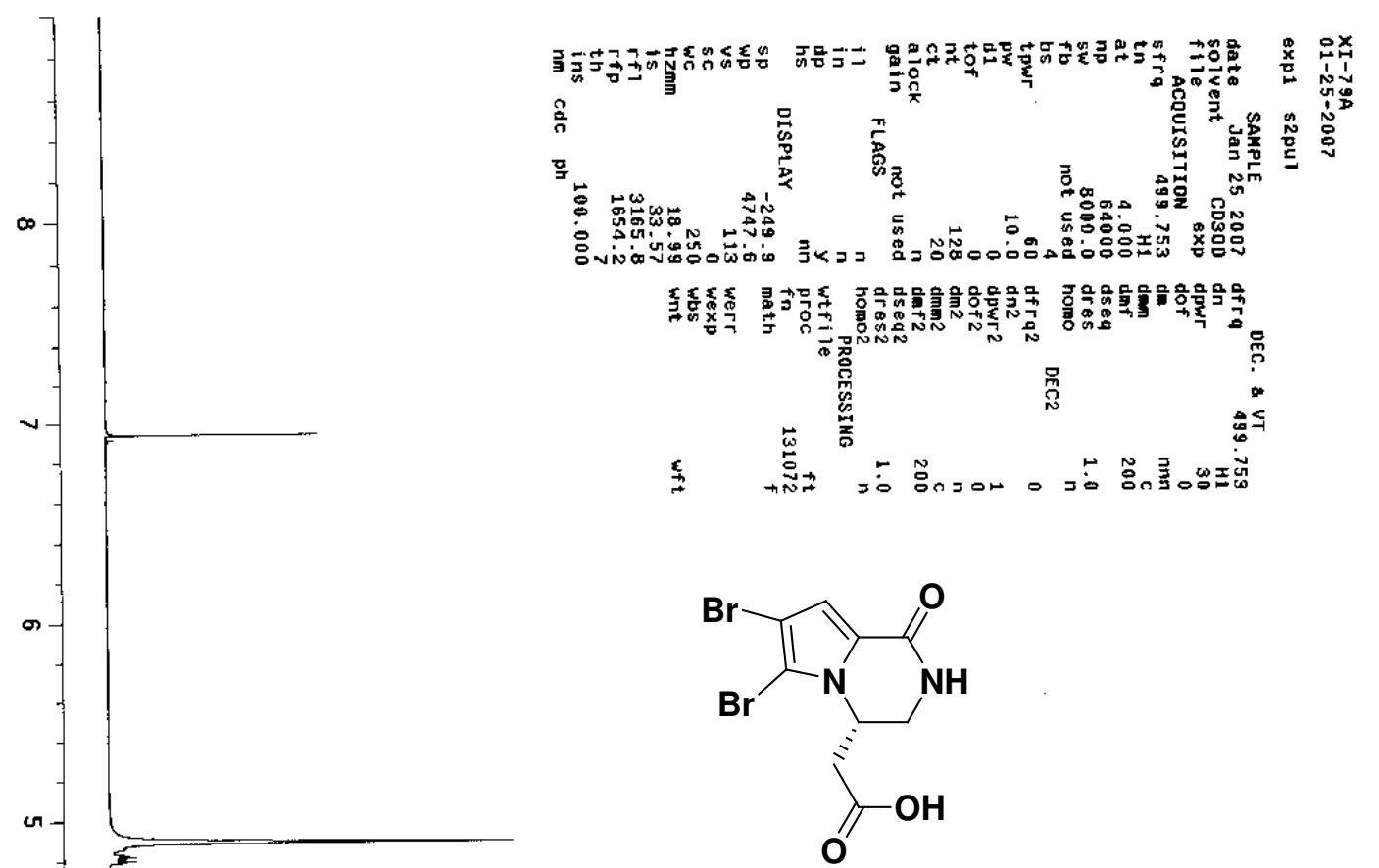

Longamide B (1)

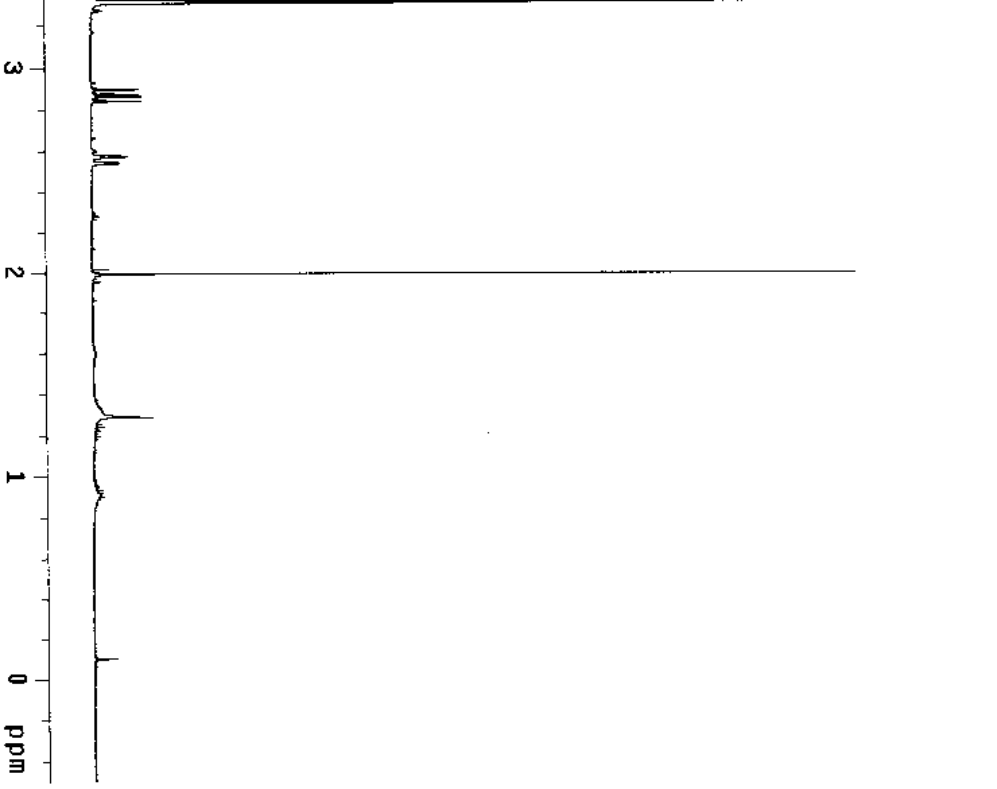




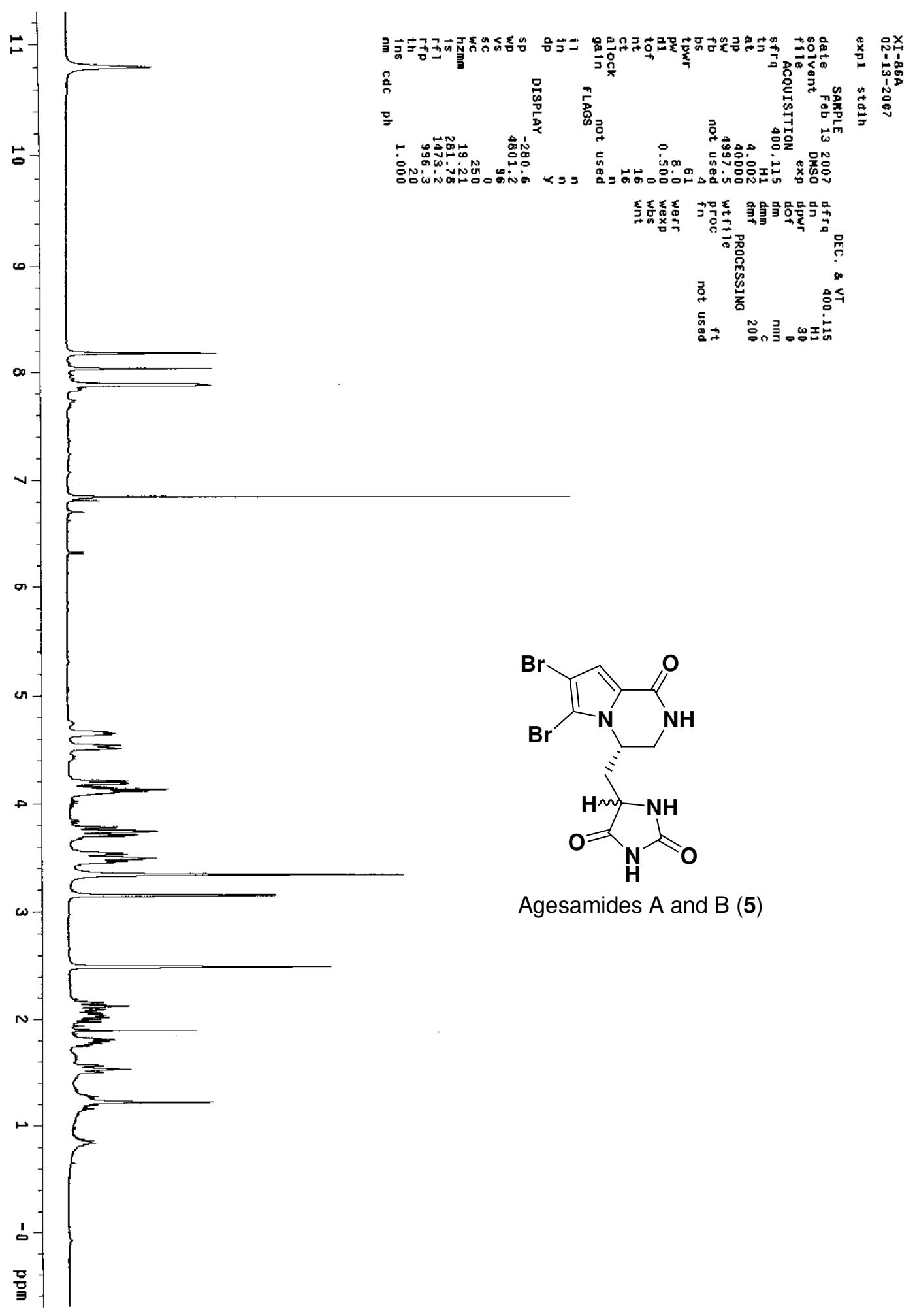




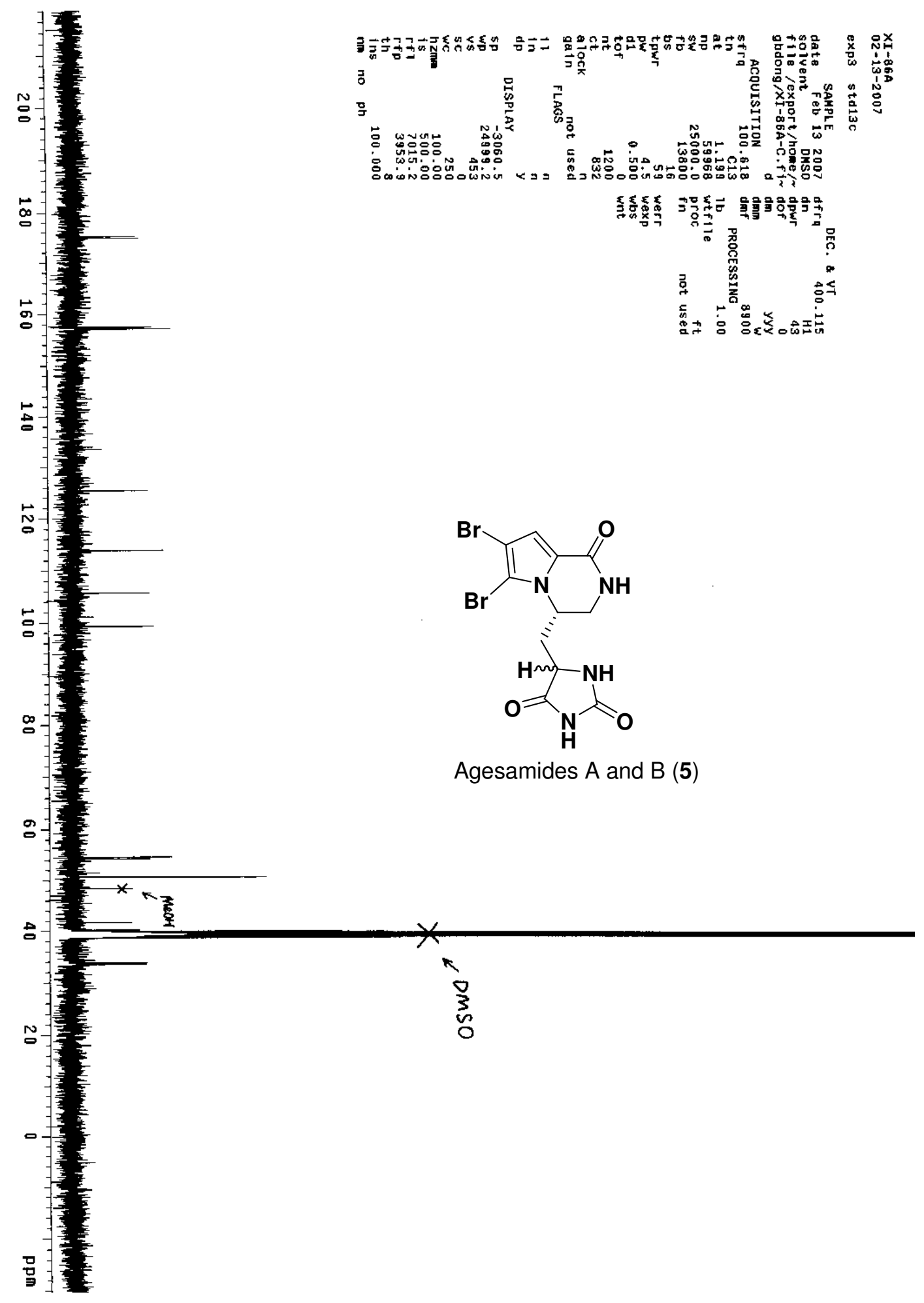

